Radiologe 2014 · 54:7-8

DOI 10.1007/s00117-013-2531-4

Online publiziert: 26. Januar 2014

c) Springer-Verlag Berlin Heidelberg 2014

C. Herold ${ }^{1} \cdot$ P. Mildenberger ${ }^{2}$

${ }^{1}$ Univ.-Klinik für Radiologie und Nuklearmedizin, Allgemeines Krankenhaus, Medizinische Universität Wien, Wien

2 Klinik und Poliklinik für Diagnostische und Interventionelle Radiologie, Universitätsmedizin der Johannes-Gutenberg-Universität Mainz, Mainz

\title{
Radiologischer Arbeitsplatz 2.0
}

Sehr geehrte Leserinnen und Leser,

die Zeit der Dunkelkammern, Entwicklungsmaschinen und Lichtkästen ist in der Radiologie - weitestgehend - vorbei. Im Verlauf der letzten Jahre ist die Radiologie vielfach aus dem „Keller“ in eine neue, zentrale Rolle für das Management der Patienten einbezogen worden. Inter- und multidisziplinäre Visiten, direkte Kommunikation und Interaktion mit unseren Kollegen aus anderen klinischen Fächern sowie zeitnahe Erfassung elektronischer Anforderungen und Übermittlung von Befunden bestimmen unseren täglichen Arbeitsablauf.

Dies hat sowohl die Gestaltung der einzelnen Arbeitsplätze, der Arbeitsmittel als auch der Arbeitsabläufe radikal verändert. Es ist mittlerweile unvorstellbar, dass eine Computertomographie eines gesamten Abdomens auf Filme ausgedruckt und am Schaukasten befundet wird, stattdessen erfordert das Bildmaterial der Schnittbildverfahren eine digitale Befundungstechnik und Archivierung. Radiologie ist heute in vielen Bereichen weitgehend computerunterstützt organisiert und kann - oder besser muss - zumindest „papierarm“ funktionieren. Damit hat unser Fach in vielerlei Beziehung eine Vorreiterrolle im Vergleich mit den anderen klinischen Partnern.

Wir Radiologen müssen uns daher mit neuen Themen befassen, für die es oft noch keine etablierten Lösungen gibt. Dies betrifft sowohl die Gestaltung des konkreten Arbeitsumfeldes wie auch die
Kommunikationswege. Der moderne radiologische Arbeitsplatz weicht in seinen Gestaltungserfordernissen dramatisch von tradierten Modellen und Designs ab. Die aktuellen Anforderungen entsprechen jenen moderner und komplexer Computerarbeitsplätze und bedingen eine dedizierte ergonomische Arbeitsplatzgestaltung. Der Beitrag von Knogler $u$. Ringl beleuchtet die Prinzipien und die Praxis moderner Arbeitsplatzgestaltung und sollte zur Pflichtlektüre nicht nur für Radiologinnen und Radiologen, sondern auch für Krankenhausadministratoren werden.

Für die Radiologie ist die strategische Gestaltung der IT-Systeme und die Unterstützung in der konkreten Applikation essenziell - hier kann ein mögliches Spannungsfeld mit zentralen IT-Einheiten oder IT-Dienstleistern entstehen oder auch eine für alle Beteiligten sinnvolle Zusammenarbeit entwickelt werden, wie der Beitrag von Buhk u. Fleischer aufzeigen kann.

Die Einbindung der Radiologie in den Klinikalltag und die Kommunikation sowohl im Gespräch mit den Patienten als auch den klinischen Partnern sind heute viel intensiver geworden, mit den entsprechenden Entwicklungen und Anpassungen der Infrastruktur und Arbeitsprozesse. Die Erwartungen und Anforderungen an die Radiologie, zum Beispiel mit einer kurzfristigen Terminierung und Befundung von Untersuchungen, definieren eine bessere Struktur von Befunden, eine zunehmende Transparenz und ein Moni- 
toring von Kennzahlen. Die Beiträge von Baumann, Hackländer und Kotter einerseits sowie Grupp u. Maurer andererseits geben hierzu Anregungen und Einblicke.

Als Radiologen werden wir auch zukünftig weiterhin flexibel Entwicklungen verfolgen müssen und wo dies sinnvoll ist, diese einbinden. Dies inkludiert beispielsweise neue Möglichkeiten der Bildverarbeitung, die Nutzung von Ressourcen im Internet, aber auch die Beschäftigung mit Fragen der Qualität und Sicherheit, denen wir uns stellen werden müssen. In diesem Zusammenhang geht der Beitrag von Sachs u. Pokieser auf Möglichkeiten und Angebote neuer digitaler Lehrund Lernmedien ein.

Wir wünschen Ihnen bei der Lektüre des nachfolgenden Hefts viel Vergnügen und hoffen, dass Sie nicht nur einen Überblick über die substanziellen Änderungen unseres Arbeitsumfeldes gewinnen, sondern auch Anregungen und praktische Anleitungen für die Gestaltung Ihres unmittelbaren Arbeitsumfeldes!

Ihre

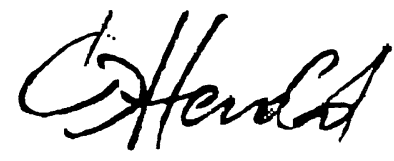

Prof. Dr. Christian Herold

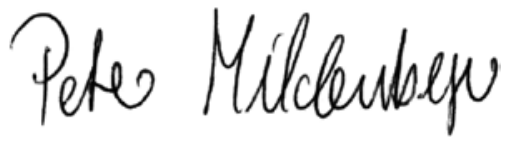

Prof. Dr. Peter Mildenberger

\section{Korrespondenzadressen}

\section{Prof. Dr. C. Herold}

Univ.-Klinik für Radiologie und Nuklearmedizin, Allgemeines Krankenhaus,

Medizinische Universität Wien,

Währinger Gürtel 18-20, 1090 Wien,

Österreich

christian.herold@meduniwien.ac.at

\section{Prof. Dr. P. Mildenberger}

Klinik und Poliklinik für Diagnostische und Interventionelle Radiologie, Universitätsmedizin der Johannes-Gutenberg-Universität Mainz, Langenbeckstraße 1, 55131 Mainz

peter.mildenberger@unimedizin-mainz.de

\section{Einhaltung ethischer Richtlinien}

Interessenkonflikt. C. Herold und P. Mildenberger geben an, dass kein Interessenkonflikt besteht.

\section{Knochentumoren}

Die pathologisch-morphologische Diagnostik von Knochentumoren basiert bevorzugt auf konventionellen Verfahren, welche heutzutage durch die Fortschritte in der Molekularpathologie ergänzt werden. Sie stellt wie keine andere Tumorentität eine Herausforderung für die interdisziplinäre Zusammenarbeit zwischen Klinikern, insbesondere Orthopäden, Radiologen und Pathologen, sowie für die weitere Therapieplanung mit den Onkologen und Strahlentherapeuten dar.

Aktuelle Entwicklungen in den verschiedenen Fachdisziplinen der Diagnostik sowie

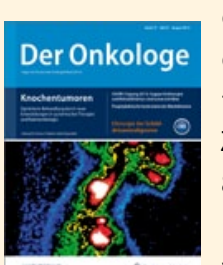
gegenwärtige Therapieoptionen von Knochentumoren werden in der Zeitschrift Der Onkologe 8/2013 mit unter anderem folgenden Themen vorgestellt:

- Pathomorphologie von Knochensarkomen

- Aktueller Stand der Molekularpathologie von Knochentumoren

- Knochentumoren aus der Sicht des Radiologen

- Operative Techniken in der Tumororthopädie

- Rolle der Strahlentherapie bei strahlensensiblen und strahlenresistenteren Knochentumoren

Bestellen Sie diese Ausgabe zum Preis von 36,- EUR zzgl. Versandkosten bei Springer Customer Service Center Kundenservice Zeitschriften

Haberstr. 7

69126 Heidelberg

Tel.: +49 6221-345-4303

Fax: +49 6221-345-4229

E-Mail: leserservice@springer.com

Suchen Sie noch mehr zum Thema? Mit e.Med, dem Online-Paket von Springer Medizin, können Sie schnell und komfortabel in über 500 medizinischen Fachzeitschriften recherchieren.

Weitere Infos unter springermedizin.de/ eMed. 